PROCEEDINGS OF THE

AMERICAN MATHEMATICAL SOCIETY

Volume 133, Number 6, Pages 1797-1804

S 0002-9939(04)07759-7

Article electronically published on December 20, 2004

\title{
LARGE AMPLITUDE PERIODIC BOUNCING FOR IMPACT OSCILLATORS WITH DAMPING
}

\author{
DINGBIAN QIAN \\ (Communicated by Carmen C. Chicone)
}

\begin{abstract}
A result of A. Lazer and P. McKenna is extended to show the existence of large amplitude periodic bouncing for a damped linear impact oscillator with multiple impacts during one period.
\end{abstract}

\section{INTRODUCTION}

Impact oscillators of the form

$$
\left\{\begin{array}{l}
x^{\prime \prime}+f\left(t, x, x^{\prime}\right)=0, \quad \text { for } x(t)>0 \\
x(t) \geq 0 \text { and } x\left(t_{0}\right)=0 \Rightarrow x^{\prime}\left(t_{0}+\right)=-x^{\prime}\left(t_{0}-\right)
\end{array}\right.
$$

serve as models of dynamical systems with discontinuities [5]. Systems of this type are special cases of vibro-impact systems [1]; they are also related to the Fermi accelerator [6, dual billiards 2] and certain models used in celestial mechanics [4. In spite of its importance, the dynamics of system (1.1) is far from being understood, even for one-degree-of-freedom linear oscillators with impacts (cf. [2, 3] 6, 7, 8, 9]).

In [7], Lazer and McKenna consider the linear impact oscillator

$$
\left\{\begin{array}{l}
x^{\prime \prime}+\delta x^{\prime}+b^{2} x=1+\varepsilon p(t), \quad \text { for } x(t)>0 \\
x(t) \geq 0 \text { and } x\left(t_{0}\right)=0 \Rightarrow x^{\prime}\left(t_{0}+\right)=-x^{\prime}\left(t_{0}-\right),
\end{array}\right.
$$

where $p$ is a $2 \pi$-periodic continuous function and $\delta=\varepsilon a(\varepsilon)$ for $a$ a continuous function such that $a(\varepsilon) \rightarrow 0$ as $\varepsilon \rightarrow 0$. They show that if $\frac{1}{2}<b<1, x_{0}$ denotes the solution of the system

$$
\left\{\begin{array}{l}
x^{\prime \prime}+b^{2} x=1 \\
x(0)=0=x(2 \pi), \quad x^{\prime}(0)=-x^{\prime}(2 \pi) \quad \text { and } \quad x(t)>0, \quad \text { for } \quad t \in(0,2 \pi),
\end{array}\right.
$$

and $h(\alpha)=\int_{0}^{2 \pi} x_{0}^{\prime}(t) p(t-\alpha) d t$ has a zero $\alpha_{0}$ such that $h^{\prime}\left(\alpha_{0}\right) \neq 0$, then there exists $\varepsilon_{0}>0$ such that system (1.2) has at least one $2 \pi$-periodic solution with an impact in $[0,2 \pi)$ whenever $|\varepsilon|<\varepsilon_{0}$.

From the viewpoint of mechanics, the result of Lazer and McKenna states that a linear oscillator whose mass is allowed to bounce elastically against a barrier will

Received by the editors February 10, 2003 and, in revised form, February 23, 2004.

2000 Mathematics Subject Classification. Primary 34C15, 34C25, 34B30.

Key words and phrases. Impact oscillator with damping, bouncing periodic solution, multiple impacts.

This work was supported by NNSF of China No.10271085 and NSF of Jiangsu Province, China No.BK2002037, 02KJB110003.

(C)2004 American Mathematical Society Reverts to public domain 28 years from publication 
undergo large amplitude $2 \pi$-periodic bouncing in the presence of a small amplitude periodic force and small viscous damping.

The restriction on the periodic force $p$ is necessary in the presence of damping. For example, if $a>0$ and $p(t) \equiv 0$, then all $2 \pi$-periodic solutions of the system

$$
\left\{\begin{array}{l}
x^{\prime \prime}+a x^{\prime}+b^{2} x=1, \quad \text { for } x(t)>0 \\
x(t) \geq 0 \text { and } x\left(t_{0}\right)=0 \Rightarrow x^{\prime}\left(t_{0}+\right)=-x^{\prime}\left(t_{0}-\right)
\end{array}\right.
$$

are constant.

Recently, Bonheure and Fabry introduced the concept of an admissible bouncing solution and obtained some existence results for the bouncing problem without damping (see [3]). But, we note that their admissible solutions may be constant. Consider, for instance, the system

$$
\left\{\begin{array}{l}
x^{\prime \prime}+\frac{x}{4}=1, \quad \text { for } x(t)>0 \\
x(t) \geq 0 \text { and } \quad x\left(t_{0}\right)=0 \Rightarrow x^{\prime}\left(t_{0}+\right)=-x^{\prime}\left(t_{0}-\right) .
\end{array}\right.
$$

Every solution with initial conditions $x(\tau)=0$ and $x^{\prime}(\tau)=v>0$ has a zero that lies to the right of $\tau$ at some $t=\tau_{1}$. It can be proved (easily) that $\tau_{1}-\tau \in(2 \pi, 4 \pi]$; thus, all $2 \pi$-periodic solutions of system (1.4) are constant. On the other hand, since $\Phi(\theta)=\int_{0}^{2 \pi}\left|\cos \frac{1}{2}(t+\theta)\right| d t=8$, Theorem 3 in 3 implies that system (1.4) has a $2 \pi$-periodic admissible solution. Hence, the existence of a periodic admissible solution does not imply the existence of a nontrivial periodic bouncing solution.

As far as we know, Lazer and McKenna's is the only result on the existence of large amplitude periodic bouncing solutions for impact oscillators with damping. It suggests a natural question: Does system (1.2) have periodic bouncing solutions with multiple impacts during one period? The purpose of this paper is to answer this question in the affirmative. We will extend Lazer and McKenna's result to the case of periodic bouncing with prescribed multiple impacts during one period. Our main result is the following theorem.

Theorem 1.1. If $\frac{1}{2}<b<1$ or $b>1$, and $m \in \mathbb{N} \cap(b, 2 b)$, then there exists a $2 \pi$-periodic solution $x_{0}(t)$ of the system

$$
\left\{\begin{array}{l}
x^{\prime \prime}+b^{2} x=1, \text { for } x(t)>0 \\
x(t) \geq 0 \text { and } x\left(t_{0}\right)=0 \Rightarrow x^{\prime}\left(t_{0}+\right)=-x^{\prime}\left(t_{0}-\right)
\end{array}\right.
$$

with exactly $m$ impacts in $[0,2 \pi)$. Moreover, using the notation in the theorem of Lazer and McKenna, if $h$ has a nondegenerate zero $\alpha_{0}$ such that $h^{\prime}\left(\alpha_{0}\right) \neq 0$, then there exists $\varepsilon_{0}>0$ such that system (1.2) has at least one $2 \pi$-periodic solution with exactly $m$ impacts in $[0,2 \pi)$ whenever $0<\varepsilon \leq \varepsilon_{0}$.

Lazer and McKenna also prove that the similar impact oscillator with negative forcing

$$
\left\{\begin{array}{l}
x^{\prime \prime}+\delta x^{\prime}+b^{2} x=-1+\varepsilon p(t), \quad \text { for } x(t)>0 \\
x(t) \geq 0 \text { and } x\left(t_{0}\right)=0 \Rightarrow x^{\prime}\left(t_{0}+\right)=-x^{\prime}\left(t_{0}-\right)
\end{array}\right.
$$

has "one impact" solutions. We will generalize their result as follows:

Theorem 1.2. If $b>0$ and $m \in \mathbb{N} \cap(b,+\infty)$, then there exists a $2 \pi$-periodic solution $x_{0}(t)$ of the system

$$
\left\{\begin{array}{l}
x^{\prime \prime}+b^{2} x=-1, \quad \text { for } x(t)>0 \\
x(t) \geq 0 \text { and } x\left(t_{0}\right)=0 \Rightarrow x^{\prime}\left(t_{0}+\right)=-x^{\prime}\left(t_{0}-\right)
\end{array}\right.
$$


with exactly $m$ impacts in $[0,2 \pi)$. Moreover, if $h(\alpha)$ has a nondegenerate zero $\alpha_{0}$ such that $h^{\prime}\left(\alpha_{0}\right) \neq 0$, then there exists $\varepsilon_{0}>0$ such that the negative impact oscillator (1.6) has at least one $2 \pi$-periodic solution with exactly $m$ impacts in $[0,2 \pi)$ whenever $0<\varepsilon \leq \varepsilon_{0}$.

In Section 2, we will prove some uniform estimates for the solutions of certain auxiliary equations. These estimates are used in Section 3 together with a limit process (motivated by the arguments in [7] and [3]) to prove the existence of bouncing solutions that satisfy appropriate Dirichlet boundary values. Finally, the main results are proved by an application of the implicit function theorem.

\section{Auxiliary equations}

Consider the solution $x(t)$ of the equation $x^{\prime \prime}+b^{2} x=1$ in the phase plane starting at $x(\tau)=0$ and $x^{\prime}(\tau)=v \neq 0$. Let $\tau_{1}$ denote the first zero of $x(t)$ with $\tau_{1}>\tau$, and let $\Delta \tau(b, v):=\tau_{1}-\tau$.

Lemma 2.1. If $v>0$, then $\Delta \tau(b, v) \in\left(\frac{\pi}{b}, \frac{2 \pi}{b}\right)$, the function $v \mapsto \Delta \tau(b, v)$ is strictly decreasing and

$$
\Delta \tau(b, v) \rightarrow \frac{\pi}{b} \quad \text { as } \quad v \rightarrow+\infty, \quad \Delta \tau(b, v) \rightarrow \frac{2 \pi}{b} \quad \text { as } \quad v \rightarrow 0^{+} .
$$

If $v<0$, then

$$
\Delta \tau(b, v)=\frac{\pi}{b}+O\left(\frac{1}{b^{2}}\right), \quad \text { as } \quad b \rightarrow \infty .
$$

Proof. We have that

$$
\begin{aligned}
x(t) & =\frac{v}{b} \sin b(t-\tau)+\frac{1}{b} \int_{\tau}^{t} \sin b(t-\tau) d s \\
& =\frac{1}{b^{2}}+\frac{\sqrt{v^{2}+\frac{1}{b^{2}}}}{b} \sin b(t-\tau+\theta(v)),
\end{aligned}
$$

where $\tan (b \theta(v))=-\frac{1}{b v}$. Also,

$$
\sin b \theta(v)=-\frac{1}{b \sqrt{v^{2}+\frac{1}{b^{2}}}}, \quad \cos b \theta(v)=\frac{v}{\sqrt{v^{2}+\frac{1}{b^{2}}}} .
$$

If $v>0$, then $b \theta(v) \in\left(-\frac{\pi}{2}, 0\right), \lim _{v \rightarrow+\infty} b \theta(v)=0$ and $\lim _{v \rightarrow 0^{+}} b \theta(v)=-\pi / 2$. From the definition of $\tau_{1}$ we have

$$
\sin b \theta(v)=\sin b(\theta(v)+\Delta \tau(b, v)) .
$$

Moreover, $b(\theta(v)+\Delta \tau(b, v)) \in\left(\pi, \frac{3}{2} \pi\right)$. Thus $b(\theta(v)+\Delta \tau(b, v))+b \theta(v)=\pi$ and therefore $\Delta \tau(b, v) \in\left(\frac{\pi}{b}, \frac{2 \pi}{b}\right)$ with $\Delta \tau(b, v)$ strictly decreasing with respect to $v$, $\lim _{v \rightarrow \infty} \Delta \tau(b, v)=\pi / 2$ and $\lim _{v \rightarrow 0^{+}} \Delta \tau(b, v)=-2 \pi / b$.

If $v<0$, then $b \theta(v) \in\left(\pi, \frac{3 \pi}{2}\right)$ and

$$
\theta(v)=\frac{\pi}{b}+O\left(\frac{1}{b^{2}}\right), \quad \text { as } \quad b \rightarrow \infty .
$$

Moreover, $b(\theta(v)+\Delta \tau(b, v))+b \theta(v)=3 \pi$, and therefore

$$
\Delta \tau(b, v)=\frac{3 \pi}{b}-2 \theta(v)=\frac{\pi}{b}+O\left(\frac{1}{b^{2}}\right), \quad \text { as } \quad b \rightarrow \infty .
$$


By Lemma [2.1] if $\frac{1}{2}<b<1$ or $b>1$, then system (1.5) has a nonconstant $2 \pi$-periodic solution. Suppose that $x_{0}(t)$ is a $2 \pi$-periodic solution of (1.5) with $m$ impacts in one period; that is, there are times $0=t_{1}<t_{2}<\cdots<t_{m}<2 \pi$ such that

$$
\begin{gathered}
x_{0}\left(t_{i}\right)=0, \quad x_{0}^{\prime}\left(t_{i}+\right)=-x_{0}^{\prime}\left(t_{i}-\right), \\
x_{0}(t)>0, \quad \text { for } t \neq t_{i}, \quad i=1,2, \cdots, m .
\end{gathered}
$$

From the symmetry of the equation, it follows that $t_{i+1}=t_{i}+\frac{2 \pi}{m}, i=1,2, \cdots, m-1$.

Let us now consider the auxiliary equation

$$
x^{\prime \prime}+g_{n}(x)=1,
$$

where

$$
g_{n}(x)= \begin{cases}b^{2} x, & x \geq 0 \\ n^{2} x, & x<0 .\end{cases}
$$

In the following, we will give some uniform estimations for the solutions of some perturbations of this system.

For $\varepsilon$ small enough and $\alpha \in \mathbb{R}$, let us consider the solution $x_{n, \varepsilon}(t)$ of the equation

$$
x^{\prime \prime}+n^{2} x=1-\varepsilon a(\varepsilon) x^{\prime}+\varepsilon p(t-\alpha)
$$

starting from $x_{n, \varepsilon}(\tau)=0, x_{n, \varepsilon}^{\prime}(\tau)=v<0$.

Lemma 2.2. Fix $\alpha \in \mathbb{R}$, and for $\varepsilon \in \mathbb{R}$ consider the solution $x_{n, \varepsilon}(t)$ of the equation

$$
x^{\prime \prime}+n^{2} x=1-\varepsilon a(\varepsilon) x^{\prime}+\varepsilon p(t-\alpha)
$$

starting from $x_{n, \varepsilon}(\tau)=0, x_{n, \varepsilon}^{\prime}(\tau)=v<0$. If $\varepsilon>0$ is sufficiently small and $\tau_{n, \varepsilon}$ denotes the first zero of this solution that is larger than $\tau$, then

$$
x_{n, \varepsilon}^{\prime}\left(\tau_{n, \varepsilon}\right)=-v+E_{1}+E_{2},
$$

where $E_{1}=O(\varepsilon)$, as $\varepsilon \rightarrow 0$ uniformly for $n \gg 1$ and $E_{2}=O\left(\frac{1}{n^{2}}\right)$, as $n \rightarrow \infty$ uniformly for $\varepsilon \ll 1$.

Proof. Note that $x_{n, \varepsilon}(t)$ has the form of

$$
\begin{gathered}
x_{n, \varepsilon}(t)=\frac{1}{n}\left[v \sin n(t-\tau)+\int_{\tau}^{t} \sin n(t-s)\left(1+\varepsilon p(s-\alpha)-\varepsilon a(\varepsilon) x_{n, \varepsilon}^{\prime}(s)\right) d s\right], \\
x_{n, \varepsilon}^{\prime}(t)=v \cos n(t-\tau)+\int_{\tau}^{t} \cos n(t-s)\left(1+\varepsilon p(s-\alpha)-\varepsilon a(\varepsilon) x_{n, \varepsilon}^{\prime}(s)\right) d s
\end{gathered}
$$

in the half-plane $x \leq 0$. For $|t-\tau| \leq 4 \pi$, Gronwall's inequality implies that $\left|x_{n, \varepsilon}^{\prime}(t)\right| \leq|2 v|$, and it follows that

$$
\left|x_{n, \varepsilon}(t)-x_{n, 0}(t)\right|+\left|x_{n, \varepsilon}^{\prime}(t)-x_{n, 0}^{\prime}(t)\right|=O(\varepsilon)
$$

as $\varepsilon \rightarrow 0$ uniformly for $n \gg 1$. Moreover, for $\tau \leq t \leq \tau+\frac{\pi}{n}+O\left(\frac{1}{n}\right)$ we have

$$
x_{n, \varepsilon}(t)=\frac{1+O(\varepsilon) M_{2}}{n^{2}}+\frac{\sqrt{v^{2}+\frac{1}{n^{2}}}}{n} \sin n(t-\tau+\theta(v)),
$$

where $M_{2}$ is a constant. Let $\Delta \tau_{n, \varepsilon}:=\tau_{n, \varepsilon}-\tau$, and note that

$$
\frac{1+O(\varepsilon) M_{2}}{n^{2}}=-\frac{\sqrt{v^{2}+\frac{1}{n^{2}}}}{n} \sin n\left(\tau_{n, \varepsilon}-\tau+\theta(v)\right)=-\frac{1}{n}\left(v \sin n \Delta \tau_{n, \varepsilon}-\frac{1}{n} \cos n \Delta \tau_{n, \varepsilon}\right) .
$$


Hence, for fixed $v<0$, we have

or

$$
\Delta \tau_{n, \varepsilon}=\frac{\pi}{n}+O\left(\frac{1+O(\varepsilon)}{n^{2}}\right), \quad \text { as } \quad n \rightarrow \infty
$$

$$
\Delta \tau_{n, \varepsilon}=O\left(\frac{1+O(\varepsilon)}{n^{2}}\right), \quad \text { as } n \rightarrow \infty .
$$

But, if (2.4) holds, then $x_{n, \varepsilon}^{\prime}(t)<0$ for $t \in\left(\tau, \tau_{n, \varepsilon}\right)$ and therefore $x_{n, \varepsilon}\left(\tau_{n, \varepsilon}\right)<$ $x_{n, \varepsilon}(\tau)=0$. This is a contradiction. So we have

$$
\Delta \tau_{n, \varepsilon}=\frac{\pi}{n}+O\left(\frac{1}{n^{2}}\right), \quad \text { as } \quad n \rightarrow \infty,
$$

uniformly for $\varepsilon \ll 1$. The estimate (2.5) implies (2.2).

The next lemma is easy; the proof is left to the reader.

Lemma 2.3. Consider the equation

$$
x^{\prime \prime}+\varepsilon a(\varepsilon) x^{\prime}+b^{2} x=1+\varepsilon p(t-\alpha),
$$

and suppose that $\bar{x}_{\varepsilon}(t)$ is its solution starting from

$$
\bar{x}_{\varepsilon}(\tau)=0, \quad \bar{x}_{\varepsilon}^{\prime}(\tau)=v
$$

for $v \in\left[\frac{1}{2} x_{0}^{\prime}(0), \frac{3}{2} x_{0}^{\prime}(0)\right]$. Then there is some $\varepsilon_{0}>0$ such that

$$
\min _{t \in[\tau, \tau+2 \pi]}\left(\left|\bar{x}_{\varepsilon}(t)\right|+\left|\bar{x}_{\varepsilon}^{\prime}(t)\right|\right) \geq d>0,
$$

for $0<|\varepsilon| \leq \varepsilon_{0}$ and $\alpha \in \mathbb{R}$, where $d$ is a constant independent of $v$, $\varepsilon$ and $\alpha$. In addition, suppose that $\tau_{\varepsilon}$ is the first zero to the right of $\tau$ and $I_{\varepsilon}:=\left(\tau, \tau_{\varepsilon}\right)$. If $\bar{x}_{0}(t)$ is the solution of $x^{\prime \prime}+b^{2} x=1$ starting from the initial value of $\bar{x}_{\varepsilon}(t)$ and $I_{0}$ denotes the interval from $\tau$ to the first zero $\tau_{1}$ of $\bar{x}_{0}(t)$ to the right of $\tau$, then

$$
\begin{gathered}
\Delta S_{\varepsilon}=\max \left\{|| \bar{x}_{\varepsilon}(t)-\bar{x}_{0}(t)|+| \bar{x}_{\varepsilon}^{\prime}(t)-\bar{x}_{0}^{\prime}(t)||: t \in I_{0} \cap I_{\varepsilon}\right\}=o(1), \\
\Delta I_{\varepsilon}=\operatorname{mes}\left(\left(I_{0} \cup I_{\varepsilon}\right) \backslash\left(I_{0} \cap I_{\varepsilon}\right)\right)=o(1),
\end{gathered}
$$

as $\varepsilon \rightarrow 0$ uniformly for $v \in\left[\frac{1}{2} x_{0}^{\prime}(0), \frac{3}{2} x_{0}^{\prime}(0)\right]$ and $\alpha \in \mathbb{R}$.

For fixed $v>0$, let $T(v)$ denote the time when the solution $x(t)$ of (1.5) starting from $x(\tau)=0, x^{\prime}(\tau)=v>0$ moves from $\tau$ to its next zero $\tau_{1}$. By Lemma 2.2 and Lemma 2.3. we know that $\forall \sigma>0$, there exist $\varepsilon_{0}>0$ and $n_{0} \in \mathbb{N}$ such that for $\varepsilon \in\left(0, \varepsilon_{0}\right]$ and $n \geq n_{0}$, the solution $x_{n, \varepsilon}(t)$ of the equation

$$
x^{\prime \prime}+\varepsilon a(\varepsilon) x^{\prime}+g_{n}(x)=1+\varepsilon p(t-\alpha)
$$

starting from $x_{n, \varepsilon}(\tau)=0, x_{n, \varepsilon}^{\prime}(\tau)=v>0$ will have its next two zeros $t_{n, \varepsilon}^{-}, t_{n, \varepsilon}^{+}$in $(\tau, T(v)+\sigma)$ and moreover, $\left|x_{n, \varepsilon}^{\prime}\left(t_{n, \varepsilon}^{ \pm}\right)-( \pm v)\right| \leq \sigma,\left(x_{n, \varepsilon}(t), x_{n, \varepsilon}^{\prime}(t)\right) \in \Gamma_{v, \sigma}$ when $t \in\left[\tau, t_{n, \varepsilon}^{-}\right]$, where

$$
\Gamma_{v, \sigma}=\left\{(x, y) \in \mathbb{R}:(v-\sigma)^{2} \leq y^{2}+b^{2} x^{2} \leq(v+\sigma)^{2}, x \geq 0\right\} .
$$

Repeating the above argument and using the continuous dependence of the solution with respect to its initial value and parameter, we can choose $\varepsilon_{0}>0$ and $n_{0} \in \mathbb{N}$ such that $x_{n, \varepsilon}(t)$ has exactly $2 m$ zeros $\left(t_{n, \varepsilon}^{1}\right)^{-}<\cdots<\left(t_{n, \varepsilon}^{m}\right)^{+}$in $(\tau, m T(v)+\sigma)$ and $\left(x_{n, \varepsilon}(t), x_{n, \varepsilon}^{\prime}(t)\right) \in \Gamma_{v, \sigma}$ when $x_{n, \varepsilon}(t) \geq 0$ and $t \in\left[\tau,\left(t_{n, \varepsilon}^{m}\right)^{+}\right]$. 
Lemma 2.4. If $\sigma>0$, then there exists an $\varepsilon_{0}>0$ and $n_{0} \in \mathbb{N}$ (both independent of $\alpha)$ such that, for $\varepsilon \in\left(0, \varepsilon_{0}\right)$ and $n \geq n_{0}$, the equation

$$
x^{\prime \prime}+\varepsilon a(\varepsilon) x^{\prime}+g_{n}(x)=1+\varepsilon p(t-\alpha)
$$

has a solution $x_{n, \varepsilon}(t)$ with $x_{n, \varepsilon}(0)=0=x_{n, \varepsilon}(2 \pi)$. Moreover, $x_{n, \varepsilon}(t)$ has exactly $2 m$ zeros $0=t_{\varepsilon, 1}^{+}<t_{\varepsilon, 1}^{-}<\cdots<t_{\varepsilon, m}^{+}<t_{\varepsilon, m}^{-}<2 \pi$ in $[0,2 \pi)$ such that

$$
\left(x_{n, \varepsilon}(t), x_{n, \varepsilon}^{\prime}(t)\right) \in \Gamma_{v, \sigma} \quad \text { for } \quad t \in \bigcap_{i=1}^{m}\left[t_{\varepsilon, i}^{+}, t_{\varepsilon, i}^{-}\right] .
$$

Proof. For $\sigma>0$, consider the solution $x_{0}^{ \pm}(t)$ of 1.5$)$ starting from

$$
x_{0}^{ \pm}(0)=0, \quad\left(x_{0}^{ \pm}\right)^{\prime}(0)=x_{0}^{ \pm}=x_{0}^{\prime}(0-) \pm \frac{\sigma}{2} .
$$

By the strict monotonicity of $T(v)$ on $v$, we know that there exists $\tilde{\sigma}<\sigma / 2$ such that $x_{0}^{+}(t)$ has $m-1$ impacts in $[0,2 \pi)$ and its $m$-th impact at $t \geq 2 \pi+\tilde{\sigma}$, while $x_{0}^{-}(t)$ has $m$ impacts in $[0,2 \pi)$ and its $m$-th impact at $t \leq 2 \pi-\tilde{\sigma}$. By the previous argument, we have $\varepsilon_{0}>0$ and $n_{0} \in \mathbb{N}$ such that for $\varepsilon \in\left(0, \varepsilon_{0}\right)$ and $n \geq n_{0}$, the solution $x_{n, \varepsilon}^{ \pm}(t)$ of (2.7) starting from

$$
x_{n, \varepsilon}^{ \pm}(0)=0, \quad\left(x_{n, \varepsilon}^{ \pm}\right)^{\prime}(0)=x_{0}^{ \pm}
$$

has exactly $2 m$ zeros in $\left(0,2 \pi \pm t_{ \pm}\right)$, where $t_{+}>\tilde{\sigma}, t_{-}<\tilde{\sigma}$. The $2 m$-th zero $t_{ \pm}^{\prime}$ of $x_{n, \varepsilon}^{ \pm}(t)$ is such that $t_{+}^{\prime}>2 \pi+\frac{1}{2} \tilde{\sigma}$ and $t_{-}^{\prime}<2 \pi-\frac{1}{2} \tilde{\sigma}$. Also, $x_{n, \varepsilon}^{ \pm}(t)$ is in $\Gamma_{x_{0}^{ \pm}, \tilde{\sigma}}$ when $x_{n, \varepsilon}^{ \pm}(t) \geq 0$, respectively. Thus, using the continuous dependence of the solution with respect to initial values, we have $v_{\varepsilon} \in\left(x_{0}^{-}, x_{0}^{+}\right)$such that the solution $x_{n, \varepsilon}(t)$ of (2.7) starting from $x_{n, \varepsilon}(0)=0$ and $x_{n, \varepsilon}^{\prime}(0)=v_{\varepsilon}$ also has $x_{n, \varepsilon}(2 \pi)=0$. Moreover, $x_{n, \varepsilon}(t)$ has exactly $2 m$ zeros $0=t_{\varepsilon, 1}^{+}<t_{\varepsilon, 1}^{-}<\cdots<t_{\varepsilon, m}^{+}<t_{\varepsilon, m}^{-}<2 \pi$ in $[0,2 \pi)$ such that

$$
\left(x_{n, \varepsilon}(t), x_{n, \varepsilon}^{\prime}(t)\right) \in \Gamma_{v, \sigma} \quad \text { for } \quad t \in \bigcap_{i=1}^{m}\left[t_{\varepsilon, i}^{+}, t_{\varepsilon, i}^{-}\right] .
$$

\section{The EXISTENCE OF BOUNCING SOLUtions}

We will use a limit process in this section. Let $\varepsilon$ be fixed. Since the sequence $\left\{x_{n, \varepsilon}(\cdot)\right\}$ obtained in Lemma 2.4 is bounded in $C^{1}[0,2 \pi]$, by passing to a subsequence if necessary, we may assume that $x_{n, \varepsilon}(t)$ converges to a continuous function $x_{0, \varepsilon}(t)$ satisfying the Dirichlet boundary condition $x_{0, \varepsilon}(0)=x_{0, \varepsilon}(2 \pi)=0$. Moreover, we will prove that $x_{0, \varepsilon}(t)$ has an elastic bounce at each of its zeros in $(0,2 \pi)$.

Assume that the $2 m$ zeros of $x_{n, \varepsilon}(t)$ in $[0,2 \pi)$ tend to

$$
0=t_{\varepsilon, 1}^{+} \leq t_{\varepsilon, 1}^{-} \leq \cdots \leq t_{\varepsilon, m}^{+} \leq t_{\varepsilon, m}^{-} \leq 2 \pi
$$

as $n \rightarrow \infty$, respectively. It is easy to see, by using (2.5), that $t_{\varepsilon, i}^{-}=t_{\varepsilon, i+1}^{+}, i=$ $1,2, \cdots, m-1, t_{\varepsilon, m}^{-}=2 \pi$. Let $t_{\varepsilon, 1}^{*}=0, t_{\varepsilon, i+1}^{*}=t_{\varepsilon, i}^{-}$for $i=1,2, \cdots, m-1$. Then $x_{0, \varepsilon}\left(t_{\varepsilon, i}^{*}\right)=0, i=1,2, \cdots, m$; and moreover, $x_{0, \varepsilon}(t) \geq 0$ for $t \in[0,2 \pi]$. For $t^{*} \in(0,2 \pi)$ and $t^{*} \neq t_{\varepsilon, i}^{*}, i=1, \cdots, m-1$, there exists $\delta_{\varepsilon}^{*}>0$ such that for $t \in\left[t^{*}-\delta_{\varepsilon}^{*}, t^{*}+\delta_{\varepsilon}^{*}\right], x_{n, \varepsilon}(t)>0$, for $n \gg 1$. Then $x_{n, \varepsilon}(t)$ satisfies (2.6) for $t \in\left[t^{*}-\delta_{\varepsilon}^{*}, t^{*}+\delta_{\varepsilon}^{*}\right]$, and it follows that $\left\{x_{n, \varepsilon}(\cdot)\right\}$ is bounded in $C^{2}\left[t^{*}-\delta_{\varepsilon}^{*}, t^{*}+\delta_{\varepsilon}^{*}\right]$. Thus, by passing to the limit as $n \rightarrow \infty$, we see that $x_{0, \varepsilon}(t)$ is a solution of (2.6) in $\left[t^{*}-\delta_{\varepsilon}^{*}, t^{*}+\delta_{\varepsilon}^{*}\right]$. Moreover, by using Lemma 2.4, we have $x_{0, \varepsilon}\left(t^{*}\right)>0, x_{0, \varepsilon}^{\prime \prime}(t)$ 
is bounded for all $t \in(0,2 \pi)$, and $t \neq t_{\varepsilon, i}^{*}$ for $i=1, \cdots, m-1$; therefore $x_{0, \varepsilon}^{\prime}(0+)$, $x_{0, \varepsilon}^{\prime}(2 \pi-)$ and $x_{0, \varepsilon}^{\prime}\left(t_{\varepsilon, i}^{*} \pm\right)$ exist and are not equal to zero. Furthermore, we have

$$
x_{0, \varepsilon}^{\prime}\left(t_{\varepsilon, i}^{*}+\right)=-x_{0, \varepsilon}^{\prime}\left(t_{\varepsilon, i}^{*}-\right), \quad i=1, \cdots, m-1 .
$$

Actually, $x_{0, \varepsilon}^{\prime}\left(t_{\varepsilon, i}^{*}+\right) \cdot x_{0, \varepsilon}^{\prime}\left(t_{\varepsilon, i}^{*}-\right)<0$. If (3.1) does not hold, we have

$$
|| x_{0, \varepsilon}^{\prime}\left(t_{\varepsilon, i}^{*}+h\right)|-| x_{0, \varepsilon}^{\prime}\left(t_{\varepsilon, i}^{*}-h\right) \| \geq d>0, \quad \text { for all } h \ll 1 .
$$

On the other hand, $x_{n, \varepsilon}(t)$ satisfies (2.7). Multiplying (2.7) by $x_{n, \varepsilon}^{\prime}(t)$ and integrating over $\left[t_{\varepsilon, i}^{*}-h, t_{\varepsilon, i}^{*}+h\right]$, for $n$ sufficiently large, we have

$$
\begin{gathered}
\frac{1}{2}\left(\left(x_{n, \varepsilon}^{\prime}\left(t_{\varepsilon, i}^{*}+h\right)\right)^{2}-\left(x_{n, \varepsilon}^{\prime}\left(t_{\varepsilon, i}^{*}-h\right)\right)^{2}\right)=-\frac{b^{2}}{2} \Delta_{1}(n, h)+\Delta_{2}(n, h) \\
-\varepsilon \int_{t_{\varepsilon, i}^{*}-h}^{t_{\varepsilon, i}^{*}+h}\left(-a(\varepsilon)\left(a(\varepsilon)\left(x_{n, \varepsilon}^{\prime}(t)\right)^{2}+p(t-\alpha) x_{n, \varepsilon}^{\prime}(t)\right)\right) d t
\end{gathered}
$$

where

$$
\begin{gathered}
\Delta_{1}(n, h)=\left(x_{n, \varepsilon}\left(t_{\varepsilon, i}^{*}+h\right)\right)^{2}-\left(x_{n, \varepsilon}\left(t_{\varepsilon, i+1}^{+}(n)\right)\right)^{2}+\left(x_{n, \varepsilon}\left(t_{\varepsilon, i}^{-}(n)\right)\right)^{2}-\left(x_{n, \varepsilon}\left(t_{\varepsilon, i}^{*}-h\right)\right)^{2}, \\
\left.\Delta_{2}(n, h)=x_{n, \varepsilon}\left(t_{\varepsilon, i}^{*}+h\right)\right)-\left(x_{n, \varepsilon}\left(t_{\varepsilon, i+1}^{+}(n)\right)\right)+\left(x_{n, \varepsilon}\left(t_{\varepsilon, i}^{-}(n)\right)\right)-\left(x_{n, \varepsilon}\left(t_{\varepsilon, i}^{*}-h\right)\right),
\end{gathered}
$$

and $t_{\varepsilon, i}^{-}(n), t_{\varepsilon, i+1}^{+}(n)$ are zeros of $x_{n, \varepsilon}(t)$. Let $n \rightarrow \infty$ to obtain

$$
\frac{1}{2}\left(\left(x_{0, \varepsilon}^{\prime}\left(t_{\varepsilon, i}^{*}+h\right)\right)^{2}-\left(x_{0, \varepsilon}^{\prime}\left(t_{\varepsilon, i}^{*}-h\right)\right)^{2}\right)=o(1), \quad \text { as } \quad h \rightarrow 0,
$$

which contradicts (3.2).

Note that $x_{0, \varepsilon}(t)$ obtained above is dependent on $\alpha$. Next we will prove that for $0<\varepsilon \leq \varepsilon_{0}$, we have $\alpha=\alpha(\varepsilon)$ such that the corresponding solution $x_{\varepsilon}(t)$ satisfies the equation $x_{\varepsilon}^{\prime}(2 \pi-)=-x_{\varepsilon}^{\prime}(0+)$. Let

$$
\begin{gathered}
G(\varepsilon, \alpha)=-a(\varepsilon) \int_{0}^{2 \pi}\left(x_{0, \varepsilon}^{\prime}(t)\right)^{2} d t+\int_{0}^{2 \pi} x_{0, \varepsilon}^{\prime}(t) \cdot p(t-\alpha) d t \\
G(0, \alpha)=\int_{0}^{2 \pi} x_{0}^{\prime}(t) \cdot p(t-\alpha) d t .
\end{gathered}
$$

Lemma2.3 and Lemma 2.4 imply that $G(\varepsilon, \alpha)$ is $C^{1}$ for $\varepsilon \in\left[-\varepsilon_{0}, \varepsilon_{0}\right]$ and $\alpha \in[0,2 \pi]$. Moreover, if $h(\alpha)=\int_{0}^{2 \pi} x_{0}^{\prime}(t) p(t-\alpha) d t$ has a simple zero $\alpha_{0}$ with $h^{\prime}\left(\alpha_{0}\right) \neq 0$, then $G\left(0, \alpha_{0}\right)=0$ and $G_{\alpha}^{\prime}\left(0, \alpha_{0}\right) \neq 0$. By the implicit function theorem, if $\varepsilon_{0}$ is sufficiently small, there is a continuous function $\alpha$ such that $G(\varepsilon, \alpha(\varepsilon))=0$ for $\varepsilon \in\left[-\varepsilon_{0}, \varepsilon_{0}\right]$.

To complete the proof for Theorem 1.1 note that $x_{0, \varepsilon}(t)$ satisfies

$$
x_{0, \varepsilon}^{\prime \prime}(t) x_{0, \varepsilon}^{\prime}(t)+\varepsilon a(\varepsilon)\left(x_{0, \varepsilon}^{\prime}(t)\right)^{2}+b^{2}\left(x_{0, \varepsilon}(t)\right) x_{0, \varepsilon}^{\prime}(t)=x_{0, \varepsilon}^{\prime}(t)+\varepsilon p(t-\varepsilon) x_{0, \varepsilon}^{\prime}(t)
$$

for $t \in[0,2 \pi]$ and $t \neq 0, t_{\varepsilon, 1}^{*}, \cdots, t_{\varepsilon, m-1}^{*}, 2 \pi$. By integrating (3.3) from 0 to $2 \pi$, we have

$$
\frac{1}{2}\left(\left(x_{0, \varepsilon}^{\prime}(2 \pi-)\right)^{2}-\left(x_{0, \varepsilon}^{\prime}(0+)\right)^{2}\right)=\varepsilon G(\varepsilon, \alpha(\varepsilon)) .
$$

As in the argument above, for $\varepsilon_{0}>0$ sufficiently small, and $0<\varepsilon \leq \varepsilon_{0}$, there is a function $\alpha$ such that $G(\varepsilon, \alpha(\varepsilon))=0$. Hence, the corresponding solution $x_{\varepsilon}(t)=$ $x_{0, \varepsilon}(t)$ satisfies $x_{\varepsilon}^{\prime}(2 \pi-)=-x_{\varepsilon}^{\prime}(0+)$, and therefore we can extend $u(t)=x_{\varepsilon}(t+\alpha(\varepsilon))$ periodically to a $2 \pi$-periodic solution of system (1.2) with exactly $m$ impacts during one period. This proves Theorem [1.1, the proof of Theorem [1.2] is similar. 


\section{ACKNOWLEDGEMENT}

The author thanks Professor Carmen C. Chicone for his kind help for the English writing of this paper.

\section{REFERENCES}

1. V.I. Babitsky, Theory of vibro-impact systems and applications. Springer-Verlag, 1998. MR1647097 (99j:70026)

2. C. Bapat, Periodic motions of an impact oscillator. Journal of Sound and Vibration 209 (1998), 43-60.

3. D. Bonheure and C. Fabry, Periodic motions in impact oscillators with perfectly elastic bounces. Nonlinearity 15 (2002), 1281-1298. MR.1912295 (2003f:34080)

4. M. Corbera and J. Llibre, Periodic orbits of a collinear restricted three body problem. Celestial Mech. Dynam. Astronom. 86 (2003), 163-183. MR1988412 (2004c:70015)

5. M. Kunze, Non-smooth Dynamical Systems. Lecture Notes in Math. 1744, Springer-Verlag, 2000. MR1789550 (2002e:34002)

6. H. Lamba, Chaotic, regular and unbounded behaviour in the elastic impact oscillator. Phys. D 82 (1995), 117-135. MR1324244 (96m:70032)

7. A. Lazer and P. McKenna, Periodic bouncing for a forced linear spring with obstacle. Diff. Int. Equs. 5 (1992), 165-172. MR 1141733 (92m:34082)

8. R. Ortega, Dynamics of a forced oscillator with obstacle. In: Variational and Topological Methods in the Study of Nonlinear Phenomena, edited by V. Benci etal., 77-89, Birkhäuser, Boston, 2001. MR:1879730 (2002h:00021)

9. L. Paoli and M. Schatzman, Resonance in impact problems. Math. Comput. Modelling 28 (1998), 385-406. MR1648765 (99g:73074)

Department of Mathematics, Suzhou University, Suzhou 215006, People's Republic OF CHINA

E-mail address: dbqian@suda.edu.cn 